\title{
Vascular calcification in patients with type 2 diabetes: the involvement of matrix Gla protein
}

\author{
Sophie Liabeuf ${ }^{1,2}$, Bourron Olivier ${ }^{3}$, Cees Vemeer ${ }^{4}$, Elke Theuwissen ${ }^{4}$, Elke Magdeleyns ${ }^{4}$, Carole Elodie Aubert ${ }^{3}$, \\ Michel Brazier ${ }^{1}$, Romuald Mentaverri ${ }^{1}$, Agnes Hartemann ${ }^{3}$ and Ziad A Massy ${ }^{1,5^{*}}$
}

\begin{abstract}
Background: Matrix Gla protein (MGP) is an important inhibitor of calcification. The objective of the present study of patients with type 2 diabetes and normal or slightly altered kidney function was to evaluate levels of inactive, dephospho-uncarboxylated MGP(dp-ucMGP) and total uncarboxylated MGP(t-ucMGP) and assess their links with biological and clinical parameters (including peripheral vascular calcification).

Methods: The DIACART study is a cross-sectional cohort study of 198 patients with type 2 diabetes and normal or slightly altered kidney function. Matrix Gla protein levels were measured with an ELISA and all patients underwent multislice spiral computed tomography scans to score below-knee arterial calcification.

Results: In the study population as a whole, the mean dp-uCMGP and t-ucMGP levels were $627 \pm 451 \mathrm{pM}$ and $4868 \pm 1613 \mathrm{nM}$, respectively. Glomerular filtration rate, age and current vitamin K antagonist use were independently associated with dp-ucMGP levels. When the study population was divided according to the median peripheral arterial calcification score, patients with the higher score displayed significantly lower t-ucMGP and significantly higher dp-ucMGP levels. Furthermore, plasma dp-ucMGP was positively associated with the peripheral arterial calcification score (independently of age, gender, previous cardiovascular disease and t-ucMGP levels).

Conclusions: High dp-ucMGP levels were independently associated with below-knee arterial calcification score in patients with type 2 diabetes and normal or slightly altered kidney function. The reversibility of the elevation of dp-ucMGP levels and the latter's relationship with clinical events merit further investigation.
\end{abstract}

Keywords: Matrix gla protein, Type 2 diabetes, Peripheral calcification

\section{Background}

Peripheral arterial disease (PAD) is a major vascular complication and the leading cause of amputation in people with diabetes. In patients with PAD, the tibial artery calcification score is a useful tool for identifying patients at high risk of amputation, since the score has greater predictive value than traditional risk factors [1]. Indeed, diabetes accelerates atherosclerosis and increases the incidence of vascular calcification (VC) [2,3]. In people with diabetes, $\mathrm{VC}$ is present in both coronary arteries and arteries of the lower limbs. Furthermore, VC is an independent predictor of cardiovascular and overall

\footnotetext{
* Correspondence: ziad.massy@apr.aphp.fr

${ }^{1}$ INSERM U1088, Jules Verne University of Picardy, F-80000 Amiens, France

${ }^{5}$ Division of Nephrology, Ambroise Paré Hospital, Paris-lle-de-France-Ouest University (UVSQ), 9 avenue Charles de Gaulle, F-92104 Boulogne-Billancourt, France

Full list of author information is available at the end of the article
}

mortalities in patients with type 2 diabetes [4]. Various epidemiologic studies have identified specific biomarkers (including osteoprotegerin, osteocalcin and others) for $\mathrm{VC}$ in this population $[5,6]$.

One of the most interesting calcification inhibitors is matrix Gla-protein (MGP), a vitamin K-dependent protein that is expressed by smooth muscle cells, fibroblasts, chondrocytes and endothelial cells in a variety of tissues (including arterial vessel wall). There is evidence to suggest that full activation of MGP requires posttranslational carboxylation and phosphorylation (Figure 1) [7,8]. In order to measure vitamin $\mathrm{K}$ status, assays were developed to measure the conformations of MGP with the least activity, i.e. forms with no posttranslational modifications (dephospho-uncarboxylated MGP (dp-ucMGP)) or at least no gammaglutamyl carboxylation (total uncarboxylated MGP (t-ucMGP)) [9]. In fact, dp-ucMGP does not possess 


\section{Posttranslational modifications in MGP}

\section{dephospho-uncarboxylated MGP (dp-ucMGP)}

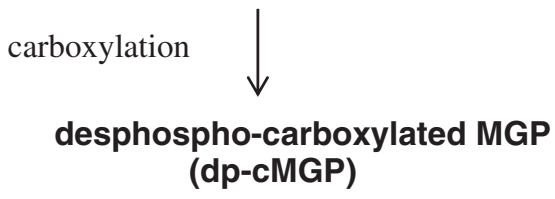

$$
\begin{array}{r}
\text { phosphorylation } \downarrow \\
\substack{\text { mature MGP } \\
(\mathrm{p}-\mathrm{cMGP})}
\end{array}
$$

Figure 1 The different forms of the matrix Gla protein (MGP). legend: MGP needs two posttranslational modifications for maturation: glutamate carboxylation and serine phosphorylation. Both modifications are only partially accomplished. Besides the non-modified form (dp-ucMGP) also partially modified species (dp-CMGP and p-UCMGP) and the fully maturated form $p$-CMGP are present in the circulation. In this paper we have tested dp-uCMGP and total ucMGP (t-ucMGP) (which consists of the sum of dp-ucMGP and p-ucMGP). Since the plasma concentration of p-ucMGP is about 10 thousand fold higher than that of dp-UCMGP, the t-uCMGP assay virtually measures p-ucMGP.

calcium-binding groups and is not retained in the vessel wall. Hence, the dp-ucMGP assay is a direct marker of vascular vitamin $\mathrm{K}$ status. In both healthy subjects and patients, poor vascular vitamin $\mathrm{K}$ status (corresponding to high circulating dp-ucMGP levels) is regarded as a risk marker for forthcoming arterial calcification. Indeed, dpucMGP was found to be associated with the severity of aortic calcification in patients with chronic kidney disease (CKD) [10]. Circulating t-ucMGP levels are at least 1000fold higher than those of dp-ucMGP, and are thought to consist mainly of phosphorylated uncarboxylated MGP (p-ucMGP) species, i.e. MGP-related antigens with between 1 and 3 high-affinity calcium-binding groups. This explains why immunohistochemical techniques invariably find ucMGP to be closely associated with calcium deposits in the vasculature; in turn, this observation is consistent with the inverse association between circulating t-ucMGP levels and the VC score.

A few studies have evaluated the association between MGP levels and VC in patients with diabetes [11-13], although the researchers used different antibodies to determine either circulating dp-ucMGP [11], t- ucMGP [12] or other conformations of ucMGP [12,14]. There are few data on (i) the levels of different MGP forms in a selected population with type 2 diabetes and (ii) the relationships between these various forms and peripheral artery calcification. This knowledge would be useful, since as Dalmeijer et al. have recently demonstrated that high dp-ucMGP levels were associated with increased cardiovascular risk (PAD and heart failure) in patients with type 2 diabetes [11]. Furthermore, Doyon et al. used a rat model of diabetes to show that the decrease in active carboxylated MGP (cMGP) levels could be due to an impairment of gammaglutamate carboxylation - suggesting that the signalling pathways involved in gammaglutamate carboxylase regulation are altered in diabetes [15].

The objective of the present study of patients with type 2 diabetes and normal or slightly altered kidney function was to evaluate (i) t-ucMGP and dp-ucMGP levels, (ii) biochemical and clinical parameters associated with differences in dp-ucMGP and t-ucMGP levels and (iii) the potential association between MGP levels and peripheral VC.

\section{Materials and methods}

Ethics statement

The study was performed in accordance with the principles of the Declaration of Helsinki and in compliance with the International Conference on Harmonization's guidelines on good clinical practice. The study protocol was approved by the local independent ethics committee (Comité de Protection des Personnes, Paris, France) prior to the initiation of any study-specific procedures. All patients were provided with full information on the study objectives and procedures and gave their written informed consent to participation.

\section{Patient selection}

In the "Diabète et Calcification Arterielle" (DIACART) cross-sectional study, 198 patients with type 2 diabetes from the Diabetology Department and the Cardiology Department at Pitié-Salpêtrière Hospital (Paris, France) were included over an 8-month period. The objective of DIACART study was to gain a better understanding of the pathophysiology of peripheral artery calcification in diabetic patients. The main inclusion criteria were (i) type 2 diabetes, with at least coronary artery disease and/or peripheral arterial occlusive disease and (ii) age $>50$ for men and $>60$ for women. The main exclusion criteria were (i) an estimated glomerular filtration rate (eGFR, calculated with the Modification of Diet in Renal Disease equation) $<30 \mathrm{ml} / \mathrm{min}$ and (ii) a history of lower limb angioplasty and/or bypass.

\section{Study protocol}

All patients were hospitalized for the day in order to perform clinical evaluations, laboratory blood tests and a multislice spiral computed tomography (CT) scan. A patient interview focused on comorbidities and the personal 
disease history. The patient's medical records were reviewed to check the information and to record vitamin $\mathrm{K}$ antagonist (VKA) use.

Previous CVD was defined as a history of any of the following events: myocardial infarction, stroke or any surgical procedures for angina or coronary disease (including percutaneous transluminal angioplasty).

\section{Laboratory tests}

Blood and urine samples were collected after an overnight fast for measurement of routine biochemistry, glycaemia, HbA1C, high-sensitivity C-reactive protein, calcium, phosphorus, 25- $\mathrm{OH}$ vitamin $\mathrm{D}$, intact parathyroid hormone (PTH), triglycerides and cholesterol.

Selected assays (including dp-ucMGP and t-ucMGP assays) were performed after the samples had been frozen, stored at $-80^{\circ} \mathrm{C}$ and thawed. A dual-antibody ELISA was used to measure dp-ucMGP levels; the capture antibody was directed against the non-phosphorylated MGP sequence 3-15 (mAb-dpMGP; VitaK BV, Maastricht, The Netherlands) and the detecting antibody was directed against the uncarboxylated MGP sequence 35-49 (mAbucMGP; VitaK BV). The same antibodies have already been used for immunohistochemical staining [16-18]. Intra-assay variability was $5,6 \%$ for dp-ucMGP and $8,9 \%$ for t-ucMGP, when inter-assay variability was $9,9 \%$ for dp-ucMGP and $11,4 \%$ for t-ucMGP. In 81 age-matched controls, the mean level of dp-ucMGP was $557 \pm 277$ pM (median: 522 pM) (measured separately in archived samples).

A competitive (single-antibody) ELISA was used to measure t-ucMGP levels, as described previously $[9,19]$. In 81 age-matched controls, the mean t-ucMGP level was $4282 \pm 1100 \mathrm{nM}$ (median: $4109 \mathrm{nM}$ ).

\section{Imaging for calculation of the below-knee arterial calcification score}

Tibial artery calcium scoring was performed after scanning with a 128-slice multidetector CT scanner (Somatom Definition Flash, Siemens Healthcare, Forchheim, Germany) in the craniocaudal direction, from the bottom of the patella down to the ankle region. Contrast agent was not used. Crosssectional slices (with: $3 \mathrm{~mm}$ ) were analyzed individually. The analysis was performed with a commercially available software package (Heartbeat CaScore, Philips Healthcare, Eindhoven, The Netherlands) by radiologists who were not aware of the results of the clinical examination or laboratory assays, On cross-sectional images, areas of calcification along below-knee arteries with a density $\geq 130$ Hounsfield units and a surface $>1 \mathrm{~mm}^{2}$ were identified automatically. The calcification scores (determined according to the method described by Agatston et al. [20]) for each of the main below-knee arteries (the distal popliteal, anterior tibial, posterior tibial and peroneal arteries) were summed to obtain the overall calcification score.

\section{Statistical analyses}

Data were expressed as the mean $\pm \mathrm{SD}$, median or frequency, as appropriate. The study patients were stratified according to the median dp-ucMGP level. This median value appears to be the best available cut off, according to the receiver operating characteristic curve for $\mathrm{VC}$ (with a sensitivity of 0.65 and a specificity of 0.70 ). Intergroup comparisons were made using a $\chi^{2}$ test for categorical variables and Student's t test or the KruskallWallis test for continuous variables. Spearman correlations were used to identify parameters correlated with dp-ucMGP levels and t-ucMGP. For parameters presenting a non-Gaussian distribution, log-normalized values were considered in tests that require normally distributed variables. Multiple linear regression analysis was used to select factors that were independently associated with dpucMGP levels. Unadjusted (Table 1) and adjusted (Table 2) logistic regression analyses were performed to evaluate the association between the peripheral calcification score (as categorized by the median) and dp-ucMGP. A p value $\leq$ 0.05 was considered to be statistically significant. All statistical analyses were performed using SPSS software (version 13.0, SPSS Inc., Chicago IL, USA) for Windows (Microsoft Corp., Redmond WA, USA).

\section{Results}

\section{Baseline characteristics}

Table 3 presents the main clinical and biochemical characteristics for the entire cohort and for the subgroups above and below the median dp-ucMGP (559.5 pM) value. Patients with higher plasma dp-ucMGP levels were significantly older, were more likely to taking VKAs and had higher body mass index, PTH levels and triglyceride levels and a lower eGFR. Patients with higher plasma t-ucMGP levels (i.e. above to the median $\mathrm{t}$ ucMGP value (4741 nM) were significantly younger, had higher body mass index, diastolic blood pressure, calcium and triglyceride levels and lower PTH levels. It is noteworthy that although only six patients were being

Table 1 Univariate logistic regression analysis: variables associated with calcification score divided by the median ( $n=198$ patients)

\begin{tabular}{lll}
\hline & Odds ratio $(95 \%$ confidence interval) & $\mathbf{p}$ \\
\hline Age & $1.07(1.03 ; 1.11)$ & $<0.0001$ \\
Ln [t-uCMGP] & $0.27(0.11 ; 0.63)$ & 0.003 \\
Ln [dp-uCMGP] & $1.88(1.21 ; 2.91)$ & 0.005 \\
Gender & $2.56(1.36 ; 4.75)$ & 0.006 \\
Previous CVD & $2.56(1.36 ; 4.75)$ & 0.004 \\
\hline
\end{tabular}

For abbreviations, please refer to Table 3.

Variables not significantly associated: diabetes duration, smoking status, VKA treatment, body mass index, SBP, DBP, GFR-MDRD, glycaemia, HbA1c, microalbuminuria, IL6, cholesterol, C-reactive protein, $25(\mathrm{OH})$ Vit D, calcium, phosphate. 
Table 2 Multivariate logistic regression analysis: variables independently associated with the calcification score divided by the median ( $\mathrm{n}=198$ patients)

\begin{tabular}{lll}
\hline & Odds ratio $(\mathbf{9 5 \%}$ confidence interval) & $\mathbf{p}$ \\
\hline Age per 1 year & $\mathbf{1 . 0 6}(\mathbf{1 . 0 2} \mathbf{1 . 1 1})$ & $\mathbf{0 . 0 0 6}$ \\
Ln [t-uCMGP] & $0.44(0.17 ; 1.15)$ & 0.092 \\
Ln [dp-uCMGP] & $\mathbf{1 . 8 8}(\mathbf{1 . 1 4 ; 3 . 1 1 )}$ & $\mathbf{0 . 0 1 4}$ \\
Male gender & $\mathbf{3 . 8 4}(\mathbf{1 . 6 6 ; 8 . 8 8 )}$ & $\mathbf{0 . 0 0 2}$ \\
Previous CVD & $\mathbf{2 . 5 6}(\mathbf{1 . 2 0} \mathbf{4 . 8 5})$ & $\mathbf{0 . 0 1 3}$ \\
\hline
\end{tabular}

Variables entered into the model: age, Ln [dp-ucMGP], Ln [t-ucMGP], gender, previous CVD.

treated with VKA, their dp-ucMGP levels were much higher than in untreated patients (the mean and median dp-ucMGP levels were $2093 \pm 1125$ pM and 1984 pM for VKA-treated patients and $581 \pm 327 \mathrm{pM}$ and $553 \mathrm{pM}$ for non-VKA-treated patients). Treatment with renin angiotensin aldosterone inhibitors was not associated with changes in levels of MGP forms or in the calcification score. We also compared patients as a function of their HbA1c levels (above vs. below 7\%) but found no difference in terms of either VC, t-ucMGP or dp-ucMGP.

\section{MGP values as a function of the calcification score}

Patients with an above-median peripheral arterial score had significantly lower t-ucMGP levels (median: $4941 \mathrm{nM}$ vs. $4550 \mathrm{nM}$, respectively; $\mathrm{p}=0.006$ ) and significantly higher dp-ucMGP levels (median: 480 pM vs. 652 pM respectively; $\mathrm{p}=0.001$ ).

\section{Associations between dp-ucMGP and calcification}

A univariate correlation analysis with plasma dp-ucMGP essentially confirmed the results as described in Table 3 (Table 4). These findings did not change when the six VKA-treated patients were excluded from the analyses. Moreover, a multivariate linear regression analysis (including all variables significantly associated with plasma dp-ucMGP levels in the univariate analyses) identified eGFR ( $p<0.0001)$, age ( $p=0.002)$ and current antivitamin $K$ use $(\mathrm{p}<0.0001)$ as independent factors.

In the present cohort, we found that age, male gender, previous CVD, and dp-ucMGP levels were positive risk factors for an elevated calcification score, whereas $t$ ucMGP appeared to protect against VC in a univariate analysis (Table 1). Furthermore, in a multivariate logistic analysis, dp-ucMGP appeared to an independent predictor of peripheral arterial calcification (independently of age, gender, previous CVD and t-ucMGP levels) (Table 2). Patients with high dp-ucMGP presented an elevated risk of $\mathrm{VC}$, independently of classical risk factors. Similar evidence was obtained after the exclusion of VKA-treated patients from the analysis.

\section{Discussion}

The present study is the first to show that in patients with type 2 diabetes and normal or slightly altered kidney function, dp-ucMGP levels (a marker for vitamin K status) are independently associated with age, eGFR and VKA treatment. More importantly, peripheral VC is associated with dp-ucMGP (independently of age, gender, previous CVD and t-ucMGP levels).

Matrix Gla protein is a strong inhibitor of $\mathrm{VC}$, as revealed by the development of massive VC in MGP knockout mice [21]. Indeed, MGP is a vitamin K-dependent inhibitor of calcium phosphate precipitation and crystal formation in the vessel wall [22]. Furthermore, it suppresses the activity of bone morphogenetic proteins 2 and 4 [22,23]. The value of MGP as a calcification biomarker has been evaluated in various cohorts, and a number of assays have been developed to measure its various conformations. In the present study, we evaluated circulating tucMGP and dp-ucMGP concentrations. We focused on dp-ucMGP because this form has low affinity for vascular calcium deposits and is secreted into the bloodstream by vascular smooth muscle cells. Moreover, dp-ucMGP is a well-known marker for vascular vitamin $\mathrm{K}$ status. High levels of dp-ucMGP have been associated with aortic calcification (independently of classical risk factors) in patients at different stages of CKD [10,24]. Similarly, the present study is the first to demonstrate that in patients with type 2 diabetes and normal or slightly altered kidney function, dp-ucMGP levels were positively associated with peripheral artery calcification (independently of age, gender, previous CVD and t-ucMGP). It is important to study VC in diabetic patients, since as calcification process could present specific features. Indeed Flammer et al. recently reported that patients with elevated HbA1c levels had a significantly higher percentage of circulating blood mononuclear cells expressing the osteoblastic marker osteocalcin. However, further research is required to establish whether these cells increase VC [25]. On the same lines, a distinct subpopulation of circulating cells expressing osteocalcin and bone alkaline phosphatase had procalcific activity in type 2 diabetic patients [26].

Indeed, the present study is the first to have evaluated the potential role of this important calcification inhibitor and its relationship with peripheral artery calcification (as evaluated with a CT-based methodology). This result is in agreement with recently published data from a prospective study of 518 patients with type 2 diabetes (mean follow-up period: 11.2 years); Dalmeijer et al. demonstrated a strong, independent relationship between high dp-ucMGP and cardiovascular risk (and PAD in particular), whereas tucMGP levels were not associated with CVD [11]. Indeed, the presence of medial calcification (which is particularly present in peripheral arteries [27]) could explain the positive correlation between dp-ucMGP levels and PAD. 
Table 3 Baseline characteristics as a function of the median plasma dp-ucMGP level

\begin{tabular}{|c|c|c|c|c|}
\hline & $\begin{array}{l}\text { All } \\
n=198\end{array}$ & $\begin{array}{l}\text { [dp-ucMGP] } \leq 559.5 \mathrm{pM} \\
\mathrm{n}=99\end{array}$ & $\begin{array}{l}\text { [dp-ucMGP] }>559.5 \mathrm{pM} \\
\mathrm{n}=99\end{array}$ & $p$ \\
\hline Age (years) & $64 \pm 8$ & $63 \pm 9$ & $66 \pm 8$ & 0.002 \\
\hline Diabetes duration (years) & $15 \pm 10$ & $14 \pm 10$ & $15 \pm 9$ & 0.358 \\
\hline Male gender n (\%) & $158(80)$ & $82(83)$ & $76(77)$ & 0.288 \\
\hline Body mass index $\left(\mathrm{kg} / \mathrm{m}^{2}\right)$ & $29 \pm 5$ & $28 \pm 5$ & $30 \pm 5$ & $<0.0001$ \\
\hline SBP $(\mathrm{mmHg})$ & $127 \pm 17$ & $126 \pm 17$ & $128 \pm 17$ & 0.377 \\
\hline $\mathrm{DBP}(\mathrm{mmHg})$ & $73 \pm 9$ & $73 \pm 9$ & $72 \pm 8$ & 0.487 \\
\hline Smoking habit n (\%) & $119(60)$ & $58(59)$ & $61(62)$ & 0.663 \\
\hline Previous CVD n (\%) & $139(70)$ & $66(67)$ & $73(74)$ & 0.277 \\
\hline \multirow[t]{2}{*}{ Glycaemia (mmol/l) } & $8.2 \pm 2.8$ & $8.0 \pm 2.6$ & $8.3 \pm 2.9$ & 0.401 \\
\hline & (7.8) & (7.6) & (8.0) & \\
\hline \multirow[t]{2}{*}{$\mathrm{HbA1c}(\%)$} & $7.8 \pm 1.5(7.5)$ & $7.7 \pm 1.4$ & $7.9 \pm 1.5$ & 0.572 \\
\hline & & $(7.5)$ & $(7.5)$ & \\
\hline GFR MDRD (mmol/l) & $80 \pm 19$ & $82 \pm 19$ & $70 \pm 19$ & $<0.0001$ \\
\hline \multirow[t]{2}{*}{ Microalbuminuria (mg/l) } & $166 \pm 840$ & $169 \pm 1132$ & $162 \pm 373$ & $<0.0001$ \\
\hline & $(23)$ & (14) & (33) & \\
\hline Calcium (mmol/l) & $2.30 \pm 0.10$ & $2.30 \pm 0.10$ & $2.34 \pm 0.10$ & 0.055 \\
\hline Phosphate $(\mathrm{mmol} / \mathrm{l})$ & $1.02 \pm 0.15$ & $1.04 \pm 0.20$ & $1.00 \pm 0.20$ & 0.164 \\
\hline Intact PTH & $54.5 \pm 27.5$ & $50.8 \pm 26.0$ & $58.5 \pm 28.9$ & 0.030 \\
\hline$(\mathrm{pg} / \mathrm{mL})$ & $(46.9)$ & $(45.2)$ & $(52.0)$ & \\
\hline \multirow[t]{2}{*}{$25(\mathrm{OH}) \mathrm{Vit} \mathrm{D}(\mathrm{ng} / \mathrm{ml})$} & $13.8 \pm 8.4$ & $14.4 \pm 8.2$ & $13.2 \pm 8.5$ & 0.175 \\
\hline & $(12.0)$ & $(13.0)$ & $(11.0)$ & \\
\hline CRP & $2.2 \pm 1.5$ & $2.2 \pm 2.6$ & $2.2 \pm 2.5$ & 0.725 \\
\hline$(\mathrm{mg} / \mathrm{l})$ & $(1.2)$ & $(1.3)$ & $(1.1)$ & \\
\hline Triglycerides & $1.6 \pm 1.1$ & $1.4 \pm 1.1$ & $1.6 \pm 0.9$ & 0.039 \\
\hline$(\mathrm{mmol} / \mathrm{L})$ & (1.3) & (1.2) & (1.4) & \\
\hline Total cholesterol (mmol/l) & $3.7 \pm 0.9$ & $3.7 \pm 0.8$ & $3.8 \pm 0.9$ & 0.283 \\
\hline LDL cholesterol & $1.9 \pm 0.7$ & $1.9 \pm 0.7$ & $2.0 \pm 0.8$ & 0.403 \\
\hline$(\mathrm{mmol} / \mathrm{L})$ & $(1.8)$ & $(1.8)$ & $(1.8)$ & \\
\hline Total cholesterol/HDL & $3.7 \pm 1.6$ & $3.5 \pm 1.1$ & $3.9 \pm 1.8$ & 0.053 \\
\hline cholesterol & $(3.5)$ & (3.3) & (3.5) & \\
\hline \multirow[t]{2}{*}{ Peripheral calcification score } & $2528 \pm 5779$ & $1609 \pm 3983$ & $3447 \pm 7040$ & $<0.0001$ \\
\hline & $(524)$ & (274) & (1096) & \\
\hline \multirow[t]{2}{*}{ t-ucMGP (nM) } & $4868 \pm 1613$ & $4815 \pm 1689$ & $4921 \pm 1539(4787)$ & 0.460 \\
\hline & $(4741)$ & $(4616)$ & & \\
\hline \multirow[t]{2}{*}{ dp-ucMGP (pM) } & $627 \pm 451$ & $342 \pm 142$ & $912 \pm 474$ & NA \\
\hline & (559) & (352) & (755) & \\
\hline VKA treatment $\mathrm{n}(\%)$ & $6(3)$ & $0(0)$ & $6(6)$ & 0.013 \\
\hline
\end{tabular}

Data are given as means \pm SD for normally distributed measures with addition of (median) for non-normally distributed values for variables with a non-Gaussian distribution or as the number (percentage) for binary variables.

SBP: systolic blood pressure; DBP: diastolic blood pressure; CVD: cardiovascular disease; $\mathrm{HbA}_{1 c}$ : haemoglobin $\mathrm{A}_{1 \mathrm{c}}$; GFR MDRD: Glomerular filtration rate calculated with the Modification of Diet in Renal Disease formula; PTH: parathyroid hormone; CRP: C-reactive protein, t-ucMGP: total uncarboxylated matrix Gla-protein; dp-ucMGP: dephospho-uncarboxylated matrix Gla-protein. VKA: vitamin K antagonist.

Only one study (in outpatients with stable CVD) reported that higher t-ucMGP levels are associated with lower mitral annular calcification (MAC) in persons without diabetes and higher MAC in persons with diabetes [12]. In the present study, a univariate analysis revealed an inverse relationship between t-ucMGP and peripheral artery calcification; however, this link was no longer significant after adjustment for dp-ucMGP. The 
Table 4 Univariate correlations: variables associated with plasma dp-ucMGP levels and t-ucMGP levels

\begin{tabular}{|c|c|c|c|c|}
\hline & \multicolumn{2}{|c|}{ dp-ucMGP levels } & \multicolumn{2}{|c|}{ t-ucMGP levels } \\
\hline & $r$ & $p$ & $r$ & $p$ \\
\hline Age & 0.294 & $<0.0001$ & -0.306 & $<0.001$ \\
\hline Diabetes duration & 0.077 & 0.278 & -0.133 & 0.062 \\
\hline Body mass index & 0.250 & $<0.0001$ & 0.291 & $<0.0001$ \\
\hline SBP & 0.027 & 0.710 & 0.171 & 0.016 \\
\hline DBP & -0.105 & 0.142 & 0.324 & $<0.0001$ \\
\hline Glycaemia & 0.046 & 0.520 & 0.130 & 0.069 \\
\hline $\mathrm{HbA1c}$ & 0.034 & 0.638 & 0.105 & 0.141 \\
\hline GFR MDRD & -0.410 & $<0.0001$ & 0.211 & 0.003 \\
\hline Microalbuminuria & 0.307 & $<0.0001$ & -0.021 & 0.766 \\
\hline Calcium & 0.145 & 0.042 & 0.255 & $<0.0001$ \\
\hline Phosphate & -0.089 & 0.214 & -0.095 & 0.182 \\
\hline Intact PTH (pg/mL) & 0.197 & 0.005 & -0.256 & $<0.0001$ \\
\hline $25(\mathrm{OH}) \mathrm{D}$ & -0.095 & 0.181 & -0.009 & 0.903 \\
\hline C-reactive protein & 0.137 & 0.055 & 0.110 & 0.120 \\
\hline Triglycerides & 0.215 & 0.002 & 0.312 & $<0.0001$ \\
\hline Total cholesterol & 0.084 & 0.240 & 0.079 & 0.268 \\
\hline Total cholesterol/HDL cholesterol & 0.188 & 0.008 & 0.202 & 0.004 \\
\hline t-ucMGP & 0.052 & 0.467 & 1.000 & $<0.0001$ \\
\hline Peripheral calcification score & 0.260 & $<0.0001$ & -0.272 & $<0.0001$ \\
\hline
\end{tabular}

For abbreviations, please refer to Table 3. $r$ =Spearman correlation coefficient.

conflicting results in these two studies might be due to differences in the calcification site evaluated (MAC versus peripheral calcification) and patient recruitment (outpatients with stable CVD versus patients in a diabetes ward). However, it appears to be important to assay for dp-ucMGP when focusing on peripheral VC, since this factor is still associated with peripheral artery calcification after multiple adjustments. Furthermore, Dalmeijer et al. reported that dp-ucMGP levels (but not t-ucMGP levels) were associated with PAD. These findings suggest that $\mathrm{VC}$ sites differ in terms of their specific features and biomarkers. This is of particular interest in the pathophysiology of $\mathrm{VC}$ in patients with diabetes, who are particularly prone to this condition. Indeed, peripheral artery calcification is very frequent in patients with diabetes and can lead to amputation.

Furthermore, we identified eGFR, age and current VKA use as independent factors for dp-ucMGP levels. It is already know that in patients with CKD, levels of inactive forms of MGP increase progressively [10]. Even when patients with normal or slightly altered kidney function are selected, eGFR is still a strong predictor of MGP levels. It is noteworthy that the link between dp-ucMGP levels and calcification appeared to be independent of kidney function in the present study.

The use of VKAs appears to be an important predictor of dp-ucMGP; the six VKA-treated patients had dp-
ucMGP levels that were around 4 times greater than in the other patients. Indeed, VKA treatment indirectly inhibits the carboxylation of vitamin K-dependent proteins via interactions with vitamin $\mathrm{K}$ epoxide reductase. Hence, VKA treatment inhibits the gamma-carboxylation of MGP and leads to an increase in inactive forms of MGP and thus prompts VC [28,29]. Indeed, epidemiological studies performed in the last decade have revealed VC in warfarintreated patients [29].

Until recently, VKAs were the only drugs for longterm treatment of thromboembolic disorders. However, novel new oral anticoagulant agents (NOACs) have now emerged (e.g. factor Xa inhibitors such as rivaroxaban, apixaban and factor IIa inhibitors such as dabigatran). Unlike VKAs, the NOACs do not interfere with vitamin $\mathrm{K}$-dependent proteins and may thus be safer with regard to VC. This advantage is particular important in diabetic populations, who are particular prone to the development of VC. However, this hypothesis needs to be confirmed in prospective trials and balanced against the efficacy and safety of NOACs.

Given that VC in general and peripheral calcification in particular are major problems in patients with diabetes, modulation of vitamin $\mathrm{K}$ status might be an interesting therapeutic option. Indeed, vitamin $\mathrm{K}$ is gaining increasing attention in terms of its therapeutic potential in $\mathrm{VC}$ [30,31]. In a recent pilot study, vitamin K supplementation 
in dialyzed patients was tested as a means of improving vitamin $\mathrm{K}$ status. Indeed, short-term supplementation with menaquinone-7 (vitamin K2) was found to reduce dpucMGP levels in haemodialysis patients [32]. It remains to be seen whether vitamin $\mathrm{K}$ supplementation could have an impact on $\mathrm{VC}$ in patients with diabetes (through evaluation in large clinical trials).

The limitation of the present study included the small sample size, the lack of evaluation of vitamin $\mathrm{K}$ intake and serum vitamin $\mathrm{K}$ levels, and the evaluation of marker levels at a single time point. The study would have been strengthened by the presence of a control group of participants with vascular calcification data, so that they could have been compared with diabetic patients. In contrast, one of the study's main strengths relates to the fact that this was the first study to concomitant evaluate $\mathrm{dp}$ ucMGP/t-ucMGP and peripheral artery calcification in patients with type 2 diabetes.

\section{Conclusion}

In patients with type 2 diabetes, with high cardiovascular risk and normal or slightly altered kidney function, elevated dp-ucMGP levels are independently correlated with the severity of peripheral artery calcification. Hence, dp-ucMGP may be a valuable biomarker in patients with diabetes. The reversibility of the elevation of dp-ucMGP levels and the latter's relationship with clinical events merit further investigation.

\begin{abstract}
Abbreviations
MGP: Matrix gla protein; Dp-uCMGP: Dephospho-uncarboxylated matrix gla protein; t-ucMGP: Total uncarboxylated matrix gla protein; p-uCMGP: Phosphorylated uncarboxylated matrix gla protein; C-MGP: Carboxylated matrix gla protein; VC: Vascular calcification; PAD: Peripheral artery disease; CKD: Chronic kidney disease; eGFR: Estimated glomerular filtration rate; $\mathrm{HbA}_{1 c}$ : Haemoglobin $\mathrm{A}_{1 \mathrm{c}}$; GFR MDRD: Glomerular filtration rate calculated with the modification of diet in renal disease formula; PTH: Parathyroid hormone; CRP: C-reactive protein; CT: Computed tomography; VKA: Vitamin k antagonist; CVD: Cardiovascular disease; SBP: Systolic blood pressure; DBP: Diastolic blood pressure.
\end{abstract}

\section{Competing interests}

The authors declare that they have no competing interests.

\section{Authors' contributions}

$\mathrm{OB}, \mathrm{AH}, \mathrm{CEA}$ had substantial contributions to conception and study design and acquisition of data. CVB, ET, EM, MB, RM, SL and ZAM had a substantial contributions to analysis and interpretation of data. SL and ZAM drafted the article. The other authors revised it critically for important intellectual content. All the authors give their final approval of the version to be published.

\section{Acknowledgments}

The authors thank Lilly Company, the University of Lausanne, and the clinical staff of the Center for Clinical Investigations Paris-Est, as well as the Diabetes and Cardiology Departments from the AP-HP Pitié-Salpêtrière Hospital in Paris for their participation in this project. This study was supported by a fund from Lilly Company. The company was involved neither in the design of the study nor in data collection. The research activities of C.E. Aubert were supported by a doctoral research scholarship from the University of Lausanne.

\section{Author details}

${ }^{1}$ INSERM U1088, Jules Verne University of Picardy, F-80000 Amiens, France.

${ }^{2}$ Clinical Research Centre, Division of Clinical Pharmacology, Amiens
University Hospital, Jules Verne University of Picardy, F-80000 Amiens, France. ${ }^{3}$ Diabetology Department, AP-HP, Pitie-Salpétrière Hospital and Pierre, Marie Curie University of Paris, F-75005 Paris, France. ${ }^{4}$ VitaK, Maastricht University, Maastricht, Netherlands. ${ }^{5}$ Division of Nephrology, Ambroise Paré Hospital, Paris-lle-de-France-Ouest University (UVSQ), 9 avenue Charles de Gaulle, F-92104 Boulogne-Billancourt, France.

Received: 7 January 2014 Accepted: 13 April 2014

Published: 24 April 2014

\section{References}

1. Guzman RJ, Brinkley DM, Schumacher PM, Donahue RM, Beavers H, Qin X: Tibial artery calcification as a marker of amputation risk in patients with peripheral arterial disease. J Am Coll Cardiol 2008, 51(20):1967-1974.

2. Kronmal RA, McClelland RL, Detrano R, Shea S, Lima JA, Cushman M, Bild $D E$, Burke GL: Risk factors for the progression of coronary artery calcification in asymptomatic subjects: results from the Multi-Ethnic Study of Atherosclerosis (MESA). Circulation 2007, 115(21):2722-2730.

3. Raggi P, Cooil B, Ratti C, Callister TQ, Budoff M: Progression of coronary artery calcium and occurrence of myocardial infarction in patients with and without diabetes mellitus. Hypertension 2005, 46(1):238-243.

4. Rossi A, Targher G, Zoppini G, Cicoira M, Bonapace S, Negri C, Stoico V, Faggiano P, Vassanelli C, Bonora E: Aortic and mitral annular calcifications are predictive of all-cause and cardiovascular mortality in patients with type 2 diabetes. Diabetes Care 2012, 35(8):1781-1786.

5. Aoki A, Murata M, Asano T, Ikoma A, Sasaki M, Saito T, Otani T, Jinbo S, Ikeda N, Kawakami M, Ishikawa SE: Association of serum osteoprotegerin with vascular calcification in patients with type 2 diabetes. Cardiovasc Diabetol 2013, 12:11.

6. Sheng L, Cao W, Cha B, Chen Z, Wang F, Liu J: Serum osteocalcin level and its association with carotid atherosclerosis in patients with type 2 diabetes. Cardiovasc Diabetol 2013, 12:22.

7. Schurgers LJ, Spronk HM, Skepper JN, Hackeng TM, Shanahan CM, Vermeer C, Weissberg PL, Proudfoot D: Post-translational modifications regulate matrix Gla protein function: importance for inhibition of vascular smooth muscle cell calcification. J Thromb Haemost 2007, 5(12):2503-2511.

8. Schurgers $\sqcup$, Uitto J, Reutelingsperger CP: Vitamin K-dependent carboxylation of matrix Gla-protein: a crucial switch to control ectopic mineralization. Trends Mol Med 2013, 19(4):217-226.

9. Cranenburg EC, Koos R, Schurgers LJ, Magdeleyns EJ, Schoonbrood TH, Landewe RB, Brandenburg VM, Bekers O, Vermeer C: Characterisation and potential diagnostic value of circulating matrix Gla protein (MGP) species. Thromb Haemost 2010, 104(4):811-822.

10. Schurgers LJ, Barreto DV, Barreto FC, Liabeuf S, Renard C, Magdeleyns EJ, Vermeer C, Choukroun G, Massy ZA: The circulating inactive form of matrix gla protein is a surrogate marker for vascular calcification in chronic kidney disease: a preliminary report. Clin J Am Soc Nephrol 2010, 5(4):568-575.

11. Dalmeijer GW, van der Schouw YT, Magdeleyns EJ, Vermeer C, Verschuren WM, Boer JM, Beulens JW: Matrix Gla Protein Species and Risk of Cardiovascular Events in Type 2 Diabetic Patients. Diabetes Care 2013, 36(11):3766-3771.

12. Parker BD, Schurgers $L$, Vermeer $C$, Schiller NB, Whooley MA, Ix JH: The association of uncarboxylated matrix Gla protein with mitral annular calcification differs by diabetes status: The Heart and Soul study. Atherosclerosis 2010, 210(1):320-325.

13. Thomsen SB, Rathcke CN, Zerahn B, Vestergaard H: Increased levels of the calcification marker matrix Gla Protein and the inflammatory markers YKL-40 and CRP in patients with type 2 diabetes and ischemic heart disease. Cardiovasc Diabetol 2010, 9:86.

14. Silaghi CN, Fodor D, Craciun AM: Circulating matrix Gla protein: a potential tool to identify minor carotid stenosis with calcification in a risk population. Clin Chem Lab Med 2013, 51(5):1115-1123.

15. Doyon M, Mathieu P, Moreau P: Decreased expression of gammacarboxylase in diabetes-associated arterial stiffness: impact on matrix Gla protein. Cardiovasc Res 2013, 97(2):331-338.

16. Schurgers LJ, Teunissen KJ, Knapen MH, Kwaijtaal M, van Diest R, Appels A, Reutelingsperger CP, Cleutjens JP, Vermeer C: Novel conformation-specific antibodies against matrix gamma-carboxyglutamic acid (Gla) protein: undercarboxylated matrix Gla protein as marker for vascular calcification. Arterioscler Thromb Vasc Biol 2005, 25(8):1629-1633. 
17. Schurgers $L$, Spronk HM, Soute BA, Schiffers PM, DeMey JG, Vermeer C: Regression of warfarin-induced medial elastocalcinosis by high intake of vitamin $\mathrm{K}$ in rats. Blood 2007, 109(7):2823-2831.

18. Shroff RC, McNair R, Figg N, Skepper JN, Schurgers L, Gupta A, Hiorns M, Donald AE, Deanfield J, Rees L, Shanahan CM: Dialysis accelerates medial vascular calcification in part by triggering smooth muscle cell apoptosis. Circulation 2008, 118(17):1748-1757.

19. Cranenburg EC, Vermeer $C$, Koos R, Boumans ML, Hackeng TM, Bouwman FG, Kwaijtaal M, Brandenburg VM, Ketteler M, Schurgers L: The circulating inactive form of matrix Gla Protein (ucMGP) as a biomarker for cardiovascular calcification. J Vasc Res 2008, 45(5):427-436.

20. Agatston AS, Janowitz WR, Hildner FJ, Zusmer NR, Viamonte M Jr, Detrano R: Quantification of coronary artery calcium using ultrafast computed tomography. J Am Coll Cardiol 1990, 15(4):827-832.

21. Luo G, Ducy P, McKee MD, Pinero GJ, Loyer E, Behringer RR, Karsenty G: Spontaneous calcification of arteries and cartilage in mice lacking matrix GLA protein. Nature 1997, 386(6620):78-81.

22. Jia G, Stormont RM, Gangahar DM, Agrawal DK: Role of matrix Gla protein in angiotensin II-induced exacerbation of vascular calcification. Am J Physiol Heart Circ Physiol 2012, 303(5):H523-H532.

23. Wajih N, Borras T, Xue W, Hutson SM, Wallin R: Processing and transport of matrix gamma-carboxyglutamic acid protein and bone morphogenetic protein-2 in cultured human vascular smooth muscle cells: evidence for an uptake mechanism for serum fetuin. J Biol Chem 2004, 279(41):43052-43060.

24. Boxma PY, van den Berg E, Geleijnse JM, Laverman GD, Schurgers LJ, Vermeer C, Kema IP, Muskiet FA, Navis G, Bakker SJ, De Borst MH: Vitamin k intake and plasma desphospho-uncarboxylated matrix Gla-protein levels in kidney transplant recipients. PLoS One 2013, 7(10):e47991.

25. Flammer AJ, GossI M, Li J, Matsuo Y, Reriani M, Loeffler D, Simari RD, Lerman LO, Khosla S, Lerman A: Patients with an HbA1c in the prediabetic and diabetic range have higher numbers of circulating cells with osteogenic and endothelial progenitor cell markers. J Clin Endocrinol Metab 2012, 97(12):4761-4768.

26. Fadini GP, Albiero M, Menegazzo L, Boscaro E, Vigili De Kreutzenberg S, Agostini C, Cabrelle A, Binotto G, Rattazzi M, Bertacco E, Bertorelle R, Biasini L, Mion M, Plebani M, Ceolotto G, Angelini A, Castellani C, Menegolo M, Grego F, Dimmeler S, Seeger F, Zeiher A, Tiengo A, Avogaro A: Widespread increase in myeloid calcifying cells contributes to ectopic vascular calcification in type 2 diabetes. Circ Res 2011, 108(9):1112-1121.

27. Johnson RC, Leopold JA, Loscalzo J: Vascular calcification: pathobiological mechanisms and clinical implications. Circ Res 2006, 99(10):1044-1059.

28. Price PA, Faus SA, Williamson MK: Warfarin causes rapid calcification of the elastic lamellae in rat arteries and heart valves. Arterioscler Thromb Vasc Biol 1998, 18(9):1400-1407.

29. Chatrou ML, Winckers K, Hackeng TM, Reutelingsperger CP, Schurgers L: Vascular calcification: the price to pay for anticoagulation therapy with vitamin K-antagonists. Blood Rev 2012, 26(4):155-166.

30. Holden RM, Morton AR, Garland JS, Pavlov A, Day AG, Booth SL: Vitamins K and D status in stages 3-5 chronic kidney disease. Clin J Am Soc Nephrol 2010, 5(4):590-597.

31. Krueger T, Westenfeld R, Ketteler M, Schurgers LJ, Floege J: Vitamin K deficiency in CKD patients: a modifiable risk factor for vascular calcification? Kidney Int 2009, 76(1):18-22.

32. Westenfeld R, Krueger T, Schlieper G, Cranenburg EC, Magdeleyns EJ, Heidenreich S, Holzmann S, Vermeer C, Jahnen-Dechent W, Ketteler M, Floege J, Schurgers $\sqcup$ : Effect of vitamin K2 supplementation on functional vitamin $\mathrm{K}$ deficiency in hemodialysis patients: a randomized trial. Am J Kidney Dis 2012, 59(2):186-195.

doi:10.1186/1475-2840-13-85

Cite this article as: Liabeuf et al: Vascular calcification in patients with type 2 diabetes: the involvement of matrix Gla protein. Cardiovascular Diabetology 2014 13:85.

\section{Submit your next manuscript to BioMed Central and take full advantage of:}

- Convenient online submission

- Thorough peer review

- No space constraints or color figure charges

- Immediate publication on acceptance

- Inclusion in PubMed, CAS, Scopus and Google Scholar

- Research which is freely available for redistribution

Submit your manuscript at www.biomedcentral.com/submit
C Biomed Central 